\title{
Secondary B-cell lymphoma diagnosed by fine-needle aspiration cytology and flow cytometry following penile carcinoma: A case report
}

\author{
HUAN WANG ${ }^{1}$, LIAN-NV QIU ${ }^{1}$, MAO WU $^{1}$, WAN-YUAN CHEN ${ }^{2}$, \\ LI-GANG REN ${ }^{3}$, XIANG-LEI HE ${ }^{2}$ and YONG-LIE ZHOU ${ }^{1}$ \\ Departments of ${ }^{1}$ Laboratory Medicine, ${ }^{2}$ Pathology and ${ }^{3}$ Urinary Surgery, \\ People's Hospital of Zhejiang Province, Hangzhou, Zhejiang 310014, P.R. China
}

Received February 1, 2015; Accepted November 10, 2015

DOI: $10.3892 / \mathrm{ol} .2016 .4279$

\begin{abstract}
The number of studies reporting lymphoma as a secondary tumor has gradually increased. However, few studies have reported that occurrence of lymphoma as a secondary tumor following treatment for penile carcinoma, particularly cases in which the lymphoma was diagnosed by fine-needle aspiration cytology and flow cytometry. The present study reports the case of a 62-year-old male patient who was troubled with frequent urination and repeated chest tightness for 5 years. The diagnosis upon admission was penile carcinoma. Two months subsequent to the tumor removal surgery, enlarged lymph nodes were extracted from the patient using fine-needle biopsy, to be analyzed using light microscopy and flow cytometry. Smear results indicated a large number of abnormal cells scattered in the right axillary lymph node. Flow cytometry immunophenotyping of fine-needle aspiration samples indicated the increased expression of cluster of differentiation (CD)79a, CD19, CD20, CD38, $\kappa$ chain and human leukocyte antigen-DR, which supported a diagnosis of B-cell lymphoma. Thus, the patient was diagnosed with B-cell lymphoma based on the results of the fine-needle aspiration biopsy and flow cytometry. The method of diagnosis and causes of therapy-related leukemia are discussed in the present report.
\end{abstract}

Correspondence to: Dr Yong-Lie Zhou, Department of Laboratory Medicine, People's Hospital of Zhejiang Province, 158 Shang-Tang Road, Hangzhou, Zhejiang 310014, P.R. China E-mail: lab_zyl@126.com

Dr Xiang-Lei He, Department of Pathology, People's Hospital of Zhejiang Province, 158 Shang-Tang Road, Hangzhou, Zhejiang 310014, P.R. China

E-mail: xianglh992004@163.com

Key words: fine-needle aspiration biopsy, flow cytometry, penile carcinoma, lymphoma

\section{Introduction}

Non-Hodgkin's lymphomas (NHLs) are a heterogeneous group of lymphoproliferative disorders originating in B, T or natural killer (NK) lymphocytes. In the United States, B-cell lymphomas represent $80-85 \%$ of all NHL cases; $15-20 \%$ of cases are T- and NK-lymphomas (1). The National Comprehensive Cancer Network guidelines considers the following common subtypes of B-cell lymphoma: Diffuse large B-cell lymphoma, $31 \%$ of cases; follicular lymphoma, $22 \%$; chronic lymphocytic leukemia/small lymphocytic lymphoma, 6\%; mantle-cell lymphoma, 6\%; mucosa-associated lymphoid tissue lymphoma, 5\% (2). Overall NHL mortality rates are $\sim 10.9$ per 100,000 individuals in one year. However, there is little information concerning the mortality rates for the specific subtypes (3).

Recent studies have demonstrated that the diagnostic accuracy of fine-needle aspiration cytology (FNAC) may improve significantly when FNAC is used in combination with flow cytometry (FCM) or immunohistochemistry (4). FNAC offers several advantages: The procedure is quick, inexpensive and the aspiration procedure exhibits very few complications (5). At present, treatment options for B-cell lymphoma differ between patients. CHOP (cyclophosphamide, doxorubicin, vincristine and prednisone) chemotherapy has been the standard treatment for patients, and subsequently rituximab was added to CHOP to improve the outcomes for patients (2), High dose therapy with autologous stem cell rescue is another alternative for relapsed or refractory patients (3).

Non-Hodgkin's lymphoma as a secondary tumor has recently gained attention following decades of neglect during the diagnosis and treatment of primary tumors (6). Krikorian et al (7) were the first to report the increase risk of secondary NHL (sNHL) in patients successfully treated for a primary tumor. Krishnan and Morgan (8) reported that the lowest occurrence rates of sNHL was $0.07 \%$, which was obtained from multinational population-based registries data in 1987, and the highest occurrence rate was 3\%, which was obtained from the Norwegian Cancer Registry Database in 2002. Usually, sNHL develops after the first 5 years of initial therapy of primary cancer. The majority of sNHL cases 


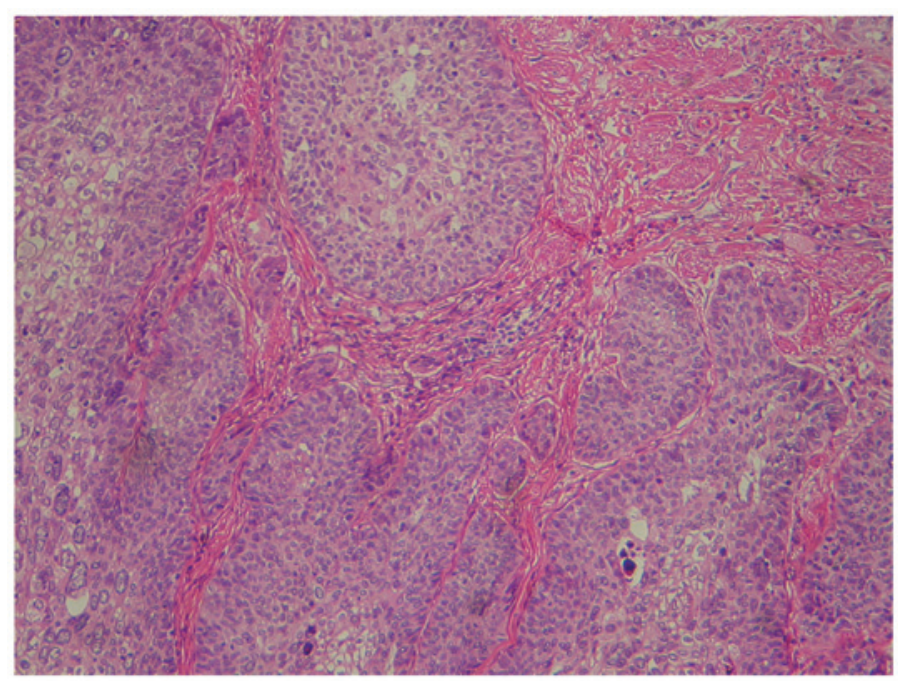

Figure 1. Histopathological analysis of the penile tumor that was removed during surgery. The penis tissue was composed of nests of heterogenous tumor cells with interstitial infiltration, and the tumor was determined to be moderately differentiated squamous cell carcinoma. The invasion depth was $\sim 7$ mm (hematoxylin and eosin staining; original magnification, $\mathrm{x} 100$ ).

develop after $\sim 5$ years of initial therapy for primary cancers. sNHLs lead to increased morbidity and mortality in these patients 8). Primary penile carcinoma has rarely been reported to lead to the development of B-cell lymphoma (9). To the best of our knowledge, the present study is the first to report a case of secondary B-cell lymphoma, diagnosed by FNAC and flow cytometry (FCM), following the treatment of penile carcinoma. Informed consent for the publication of this data was obtained from the patient.

\section{Case report}

The patient, a 62-year-old man, had suffered from frequent urination, repeated chest tightness, and weakness for 5 years. The situation worsened 1 month prior to his admission to the People's Hospital of Zhejiang Province (Hangzhou, China) in May 2014. The patient was diagnosed with hyperplasia of the prostate gland and cardiomyopathy.

A physical examination of the patient revealed a long foreskin and a thick and swollen cauliflower-like mass (diameter, $3 \mathrm{~cm}$ ) on the head of the penis. The right inguinal lymph nodes were swollen but painless. A preoperative biopsy of the lymph nodes indicated squamous cell carcinoma. The patient underwent a penis and scrotal skin excision, and the postoperative pathological diagnosis revealed a moderately differentiated squamous cell carcinoma (Fig. 1).

In July 2014, the patient returned to the People's Hospital of Zhejiang Province with a swollen and painful cheek. Upon physical examination, the superficial lymph nodes on the left neck, and right axillary and inguinal regions all demonstrated severe swelling, high activity and slight tenderness. Additional auxiliary examinations were performed. B-mode ultrasonography revealed that the lymph nodes of the bilateral axilla, groin and neck were enlarged. A chest computed tomography (CT) scan revealed multiple masses at the mediastinum, hilum of the right lung and the right axilla. In addition, bilateral lung lesions, a tubercle at the anterior side of the right upper lobe, which does not rule out lymph node infiltration, a calcified lesion at the apex of the right lung, small levels of bilateral pleural effusion, and pericardial effusion were identified. The laboratory examination data were as follows: White blood cell count, $4.64 \times 10^{9}$ cells/ 1 (normal range, $4-10 \times 10^{9}$ cells/1), including $77.1 \%$ neutrophils (normal range, 50-75\%), 14.8\% lymphocytes (normal range, 20-40\%), 6.1\% monocytes (normal range, 2-12\%), 1.8\% eosinophils (normal range, $0.5-5 \%$ ) and $0.2 \%$ basophils (normal range, 0-2\%); hemoglobin, $106 \mathrm{~g} / \mathrm{l}$ (normal range, 120-160 g/l);

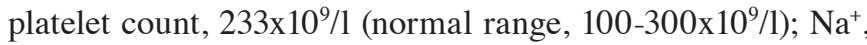
$3.81 \mathrm{mmol} / \mathrm{l}$ (normal range, 135-145 mmol/l); $\mathrm{K}^{+}, 137 \mathrm{mmol} / \mathrm{l}$ (normal range, 3.5-5.5 mmol/l); $\mathrm{Cl}^{-}, 96.7 \mathrm{mmol} / 1$ (normal range, 96-108 $\mathrm{mmol} / \mathrm{l}$ ); total protein, $49.5 \mathrm{~g} / 1$ (normal range 65-85 g/l); albumin, $27.1 \mathrm{~g} / 1$ (normal range, 40-55 g/l); globulin, $22.4 \mathrm{~g} / 1$ (normal range, 20-40 g/l); aspartate transaminase, 20 units/l (normal range, 0-50 units/l); alanine transaminase, 28 units/1 (normal range, 10-52 units/l); alkaline phosphatase, 93 units/1 (45-125 units/1); blood urea nitrogen, $7.75 \mathrm{mmol} / 1$ (normal range, 2.85-7.14 mmol/1); creatinine, $93.3 \mu \mathrm{mol} / 1$ (normal range, 44-133 $\mu \mathrm{mol} / \mathrm{l}$ ); and uric acid, $774 \mu \mathrm{mol} / 1$ (normal range, $210-440 \mu \mathrm{mol} / \mathrm{l}$ ). A routine urine test revealed no abnormality; however, levels of tumor markers in the serum increased, including the levels of carbohydrate antigen 125 at 52.4 units $/ \mathrm{ml}$ (normal range, 0-35 units $/ \mathrm{ml}$ ) and cytokeratin 19 at $5.9 \mathrm{ng} / \mathrm{ml}$ (normal range, $0-3.8 \mathrm{ng} / \mathrm{ml}$ ). The other laboratory findings were also normal. A bone marrow smear analysis revealed the presence of hyperplastic myelocytes, with a large number of myelocyte and metamyelocyte cells and a low number of segmented granulocytes with toxic particles (data not shown). FNAC was performed on the lymph node of the present patient. The standard surgical procedure is as follows: Firstly, the skin above the mass to undergo biopsy is swabbed with an antiseptic solution; the skin, underlying fat and muscle may be numbed with a local anesthetic, although this is often not necessary with superficial masses. Secondly, a needle of extremely fine diameter is passed into the mass and withdrawn several times for sampling of cells. Finally, these cells are used in a smear and examined under a microscope or rendered into a suspension used for flow cytometry (10). A large 
A

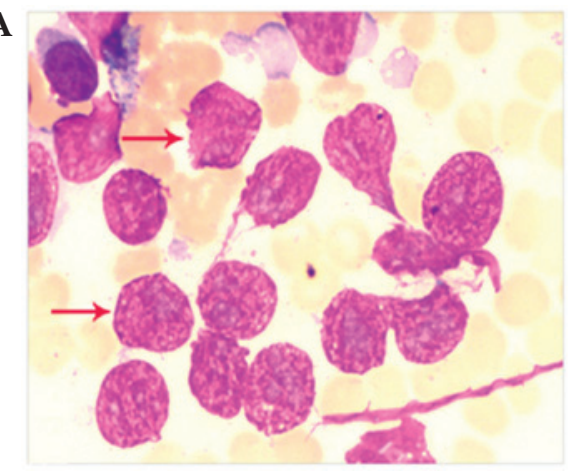

B

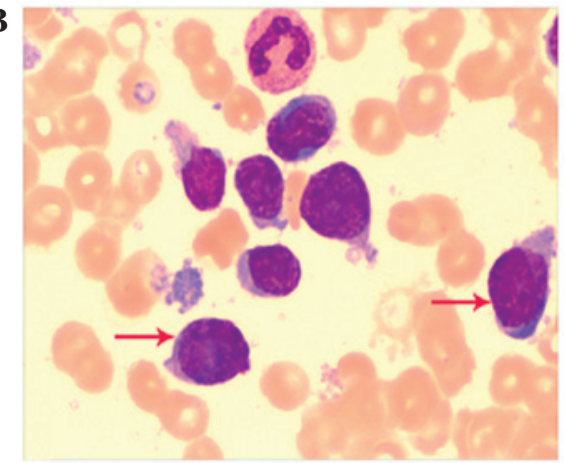

Figure 2. Smears of the lymph node biopsy samples obtained by fine-needle aspiration. (A) Large numbers of various sized and bare nuclei cells were present in the smear, and chromatin of the cells was dense (arrows). (B) Few round and large malfored cells were oberved in the smear, the cytoplasms were smaller with no particles. Nuclear size was irregular and the nucleoplasm ratio was high. Chromatin was loose and colored blue and nucleolus were faintly visible (arrows). (Wright staining; original magnification, x1,000).
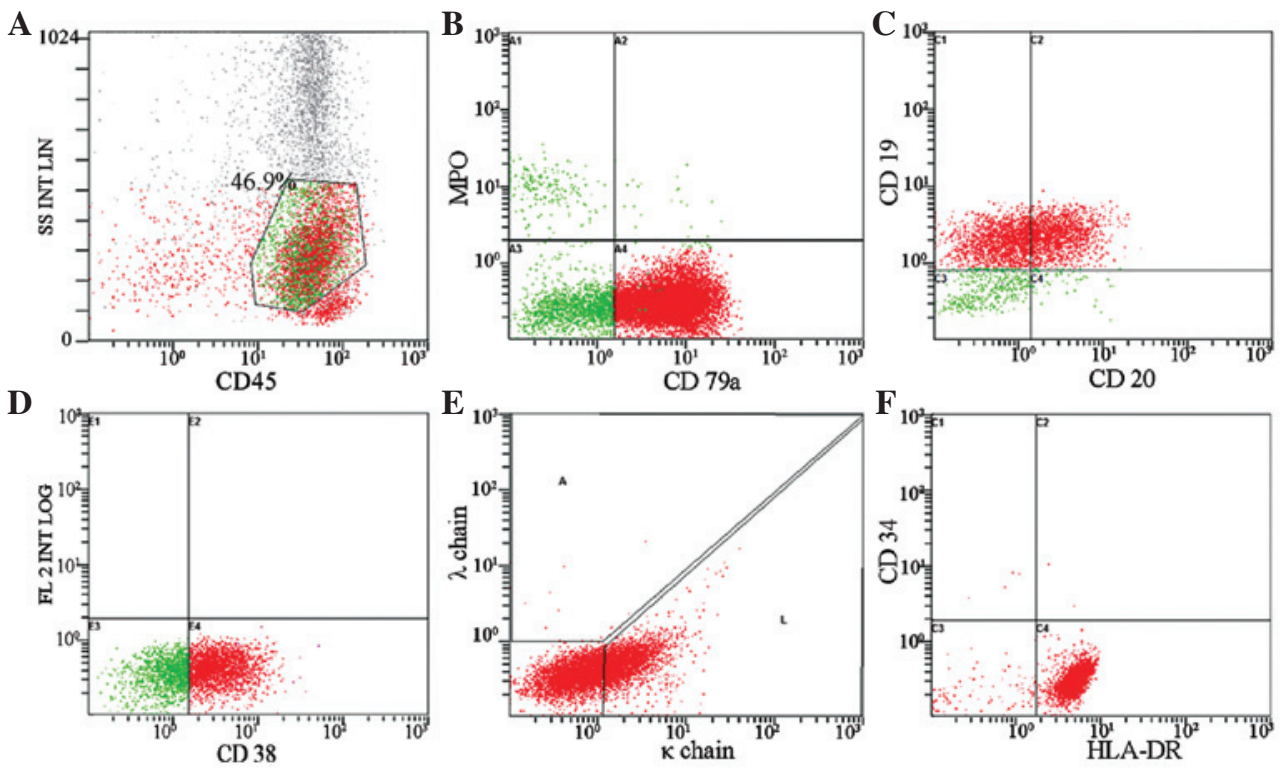

Figure 3. Flow cytometry immunophenotyping of the fine-needle aspiration sample of the lymph node. (A) Abnormal lymphocyte cell population demonstrated by forward and side scattered light. Increased expression of (B) CD79a, (C) CD20, (D) CD38, (E) $\kappa$ chain and (F) HLA-DR. CD, cluster of differentiation; HLA-DR, human leukocyte antigen-DR; SS INT LIN, side scatter integral linear; MPO, myeloperoxidase; FL2 INT LOG, fluorescence channel 2 integral logarithmic.

number of abnormal and bare nuclei cells were distributed in the current smears obtained using FNAC, which were indicative of lymphoma cells (Fig. 2). In the present study, immunophenotyping of the aspirate obtained from FNAC revealed the following data, which supported the concept of a B-cell origin: Cluster of differentiation (CD) $3^{+}, 6.8 \%$; $\mathrm{CD}^{+} \mathrm{CD}^{+}, 1.9 \%$; $\mathrm{CD}^{+} \mathrm{CD}^{+}, 3.2 \%$; natural killer, $2.7 \%$; $\mathrm{CD}^{+} 9^{+}, 88.1 \%$; $\mathrm{CD}^{+} 0^{+}$, $0.2 \%$; CD20, $42.7 \%$; CD79a, 84.8\%; CD38, 64.9\%; CD23 ${ }^{+}$, $6.5 \%$; $\kappa$ chain, $31.0 \%$; and $\lambda$ chain, $1.4 \%$ (Fig. 3 ).

\section{Discussion}

The number of studies reporting lymphoma as a secondary cancer has gradually increased, and the condition has been described following a number of types of primary tumor, including neuroblastoma (11), breast cancer (12), follicular thyroid carcinoma (13) and parotid gland cancer (14). There are a number of potential mechanisms acting in the pathogenesis of NHL, which may be associated with radiation, cytotoxic drugs and immunosuppression (8). In the present report, a patient with penile cancer developed enlarged lymph nodes two months subsequent to penile cancer surgery. The recurrence of enlarged lymph nodes at the left neck, and right axillary and inguinal regions was the major reason for the readmission of the patient to the hospital. The tissues specimens that had been previously diagnosed with penile cancer were immunohistochemically examined and markers for B-cell lymphoma (CD19, CD20, CD22 and CD79a) were negative (data not shown); therefore, the possibility of penile cancer and B-cell lymphoma appearing at the same time was eliminated, and the B-cell lymphoma was concluded to be a secondary tumor that developed subsequent to penile cancer.

Immunophenotypic analysis may be performed using flow cytometry or immunohistochemistry, the choice depends on the antigens as well as the expertise and resources available to the hematopathologist. Immunohistochemistry has certain limitations on the diagnosis of certain tumors, including in cases where the lymph node is not easily accessible, such as 
Hodgkin's lymphoma (15). FNAC is a simple, safe, minimally invasive and fast technique that is well tolerated by patients. In addition, the single cell suspension that is suitable for FCM analysis is easy to prepare (16). FCM is a rapid and sensitive multi-parameter analysis technique, which is important in the diagnosis of patients with lymph node enlargement (17). In the present study, a fine-needle aspiration biopsy of the lymph node was performed and FCM analyses were conducted. Immunophenotyping by FCM revealed increased expression of CD79a, CD19, CD20 and $\kappa$ chain, and a diagnosis of B-cell lymphoma was confirmed.

The present study demonstrates that the combination of FNAC and FCM with lymph node aspiration analysis may improve the accuracy and sensitivity of lymphoma diagnoses. Therefore, the combined technique used in the present study may be used as the routine method in lymphoma diagnosis (18). The application of this technology in clinical practice will contribute to the earlier diagnosis and treatment for patients with secondary lymphoma.

\section{Acknowledgements}

The present study was supported by the National Natural Science Foundation of China (grant no. 81301406) and the Provincial Natural Science Foundation of Zhejiang (grant nos. LQ13H190005 and LQ12H16019).

\section{References}

1. Jemal A, Siegel R, Ward E, Hao Y, Xu J, Murray T and Thun MJ: Cancer Statistics, 2008. CA Cancer J Clin 58: 71-96, 2008.

2. NCCN: The National Comprehensive Cancer Network Clinical Practice Guidelines in Oncology: Non-Hodgkin's Lymphomas. Version 2.2011.

3. Howlader N, Morton LM, Feuer EJ, Besson C and Engels EA: Contributions of subtypes of non-Hodgkin lymphoma to mortality trends. Cancer Epidemiol Biomarkers Prev 25: 174-179, 2016.

4. Zeppa P, Marino G, Troncone G, Fulciniti F, De Renzo A, Picardi M, Benincasa G, Rotoli B, Vetrani A and Palombini L: Fine-needle cytology and flow cytometry immunophenotyping and subclassification of non-Hodgkin lymphoma: A critical review of 307 cases with technical suggestions. Cancer 102: 55-65, 2004.
5. Silas OA, Ige OO, Adoga AA, Nimkur LT and Ajetunmobi OI: Role of fine needle aspiration cytology (FNAC) as a diagnostic tool in paediatric head and neck lymphodenopathy. J Otol Rhinol 4: 2015.

6. El-Mallawany NK and Cairo MS: Advances in the diagnosis and treatment of childhood and adolescent B-cell non-Hodgkin lymphoma. Clin Adv Hematol Oncol 13: 113-123, 2015

7. Krikorian JG, Burke JS, Rosenberg SA and Kaplan HS: Occurrence of non-Hodgkin's lymphoma after therapy for Hodgkin's disease. N Engl J Med 300: 452-458, 1979.

8. Krishnan B and Morgan GJ: Non-Hodgkin lymphoma secondary to cancer chemotherapy. Cancer Epidemiol Biomarkers Prev 16: 377-380, 2007.

9. Sonpavde G, Pagliaro LC, Buonerba C, Dorff TB, Lee RJ and Di Lorenzo G: Penile cancer: Current therapy and future directions. Ann Oncol 24: 1179-1189, 2013.

10. Berner A, Lund-Iversen M, Nesland JM: Fine needle aspirations in oncology. Arkh Patol 73: 21-26, 2011.

11. Imashuku S, Hibi S, Kosaka K, Tabata Y, Naya M, Hohjo M and Todo $S$ : Secondary lymphoid malignancy in two children with neuroblastoma. Med Pediatr Oncol 27: 54-56, 1996.

12. Fritzsche FR, Pahl S, Petersen I, Burkhardt M, Dankof A, Dietel M and Kristiansen G: Anaplastic large-cell non-Hodgkin's lymphoma of the breast in periprosthetic localisation 32 years after treatment for primary breast cancer - A case report. Virchows Arch 449: 561-564, 2006.

13. Lu J, Frater JL, Kreisel FH, Marcus JN and Hassan A: Secondary lymphoma involving metastatic follicular thyroid carcinoma to the skull: A unique example of tumor-to-tumor metastasis. Head Neck Pathol 2: 209-212, 2008.

14. Williams-Smith L, Gupta R and Osborne RF: Secondary lymphoma of the parotid gland: Clinical experience. Ear Nose Throat J 92: 63, 2013.

15. Dunphy CH: Applications of flow cytometry and immunohistochemistry to diagnostic hematopathology. Arch Pathol Lab Med 128: 1004-1022, 2004.

16. Lieu D: Cytopathologist-performed ultrasound-guided fine-needle aspiration and core-needle biopsy: A prospective study of 500 consecutive cases. Diagn Cytopathol 36: 317-324, 2008.

17. Mathiot C, Decaudin D, Klijanienko J, Couturier J, Salomon A, Dumont J and Vielh P: Fine-needle aspiration cytology combined with flow cytometry immunophenotyping is a rapid and accurate approach for the evaluation of suspicious superficial lymphoid lesions. Diagn Cytopathol 34: 472-478, 2006.

18. Jorgensen JL: State of the Art Symposium: Flow cytometry in the diagnosis of lymphoproliferative disorders by fine-needle aspiration. Cancer 105: 443-451, 2005. 